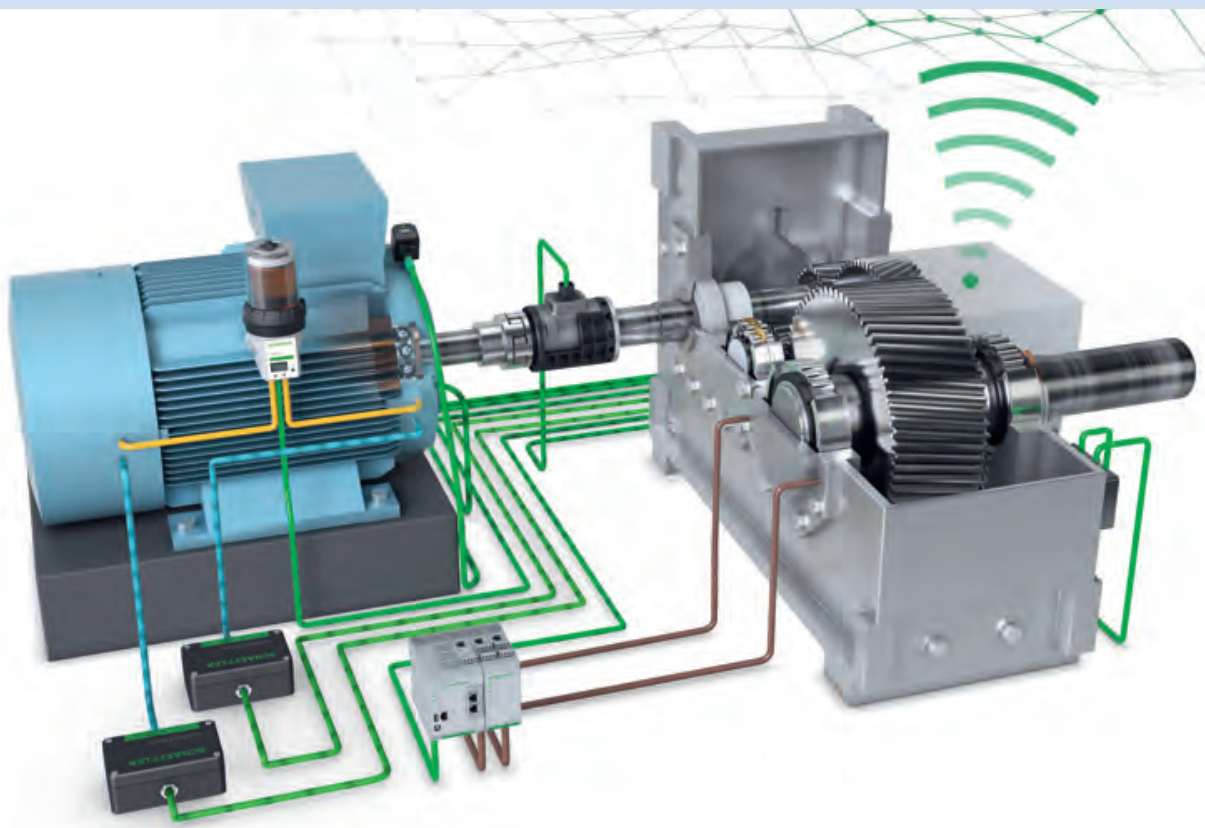

\title{
3. VDI-Fachtagung
} Schwingungen 2021

Würzburg, 16. und 17. November 2021 


\section{VDI-BERICHTE}

Herausgeber:

VDI Wissensforum GmbH 
Bibliographische Information der Deutschen Nationalbibliothek

Die Deutsche Nationalbibliothek verzeichnet diese Publikation in der Deutschen Nationalbibliographie;

detaillierte bibliographische Daten sind im Internet unter www.dnb.de abrufbar.

Bibliographic information published by the Deutsche Nationalbibliothek (German National Library)

The Deutsche Nationalbibliothek lists this publication in the Deutsche Nationalbibliographie (German National Bibliography);

detailed bibliographic data is available via Internet at www.dnb.de.

\section{(c) VDI Verlag GmbH · Düsseldorf 2021}

Alle Rechte vorbehalten, auch das des Nachdruckes, der Wiedergabe (Photokopie, Mikrokopie), der Speicherung in Datenverarbeitungsanlagen und der Übersetzung, auszugsweise oder vollständig.

Der VDI-Bericht, der die Vorträge der Tagung enthält, erscheint als nichtredigierter Manuskriptdruck.

Die einzelnen Beiträge geben die auf persönlichen Erkenntnissen beruhenden Ansichten und Erfahrungen der jeweiligen Vortragenden bzw. Autoren wieder. Printed in Germany. 


\section{Inhalt}

Vorwort. ...........................

\section{- Plenarvortrag}

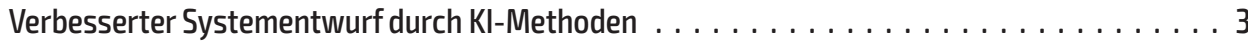

D. Bestle, Brandenburische Technische Universität Cottbus-Senftenberg, Cottbus

\section{Condition Monitoring und KI-Methoden}

Stand und Tendenzen der Normung zum Thema Schwingungsüberwachung . . . . . . . . . 15

J. Kolerus, Technische Universität Wien;

E. Becker, Fluke Deutschland GmbH, Ismaning

Praxiserfahrungen mit der Schwingungsbewertung von Industriegetrieben nach DIN ISO 10816-3 und DIN ISO 20816-9. . . . . . . . . . . . . . . . . . . . . . . . . . . . .29 J. Deckers, Flender GmbH, Voerde

Flächendeckendes Condition Monitoring - Wirtschaftlich und flächendeckend - geht das? . . 41 S. Mergler, Schaeffler Technologies AG \& Co. KG, Schweinfurt;

H.-W. Keßler, Schaeffler Monitoring Services GmbH, Herzogenrath

\section{$>$ Condition Monitoring}

Schwingungsbasierte Fehlererkennung und Schadensdetektion an Getrieben durch Einbindung von Methoden des Machine Learning . . . . . . . . . . . . . . . 53 T. König, R. Bader, M. Kley, Hochschule Aalen, Institut für Antriebstechnik

Condition Based Maintenance (CBM) an Lokomotiven über Fahrmotor Sweeps Werkstatttaugliches CBM Verfahren für Lokomotiven. . . . . . . . . . . . . . .67 W. Breuer, W. Rulka, Siemens Mobility GmbH SMO RS LMC EN LN SC, München

Monitoring von geschraubten Verbindungen mit elektromechanischen Impedanzspektren . . 77 A.-L. Dreisbach, C.-P. Fritzen, Arbeitsgruppe Technische Mechanik (ATM), Universität Siegen

Entwicklung und Validierung einer Methode zur Ermittlung der minimalen Performanceanforderungen an Sensoren für die schwingungsbasierte Zustandsüberwachung . . . . . . . .89 M. Bauer, D. Proksch, J. Kopetschek, F. Wagner, M. Kley, Hochschule Aalen, Institut für Antriebstechnik, Aalen 


\section{> Simulation und experimentelle Validierung}

Scale-Up-Verfahren zur Ermittlung der Eigenfrequenzen geometrisch ähnlicher Strukturen von Baureihen. . . . . . . . . . . . . . . . . . . . . . . . . . . . . . . 105 M. Klöcker, H. Hallmann, S. Handl, Institut für Produktentwicklung und Konstruktionstechnik, Technische Hochschule Köln;

T. Weber, Pollrich GmbH, Siegen;

A. Osteresch, Vibration Analyst, Rhede/Ems

Experimentelle Erkennung von Kopplungen in verteiltparametrischen Systemen. . . . . . . . . 119 G. Mikota, Johannes Kepler Universität Linz, Österreich

Charakterisierung der Wechselwirkung der Lagerstelle und Aggregatgehäuse auf die Strukturdynamik von Kunststoffbauteilen . . . . . . . . . . . . . . . . . . . . . . . . . . 129

A. Kriwet, K. Raschke, Mercedes-Benz AG, Stuttgart;

M. Stommel, Leibniz-Institut für Polymerforschung e.V., Dresden

Viskoelastische Dämpfungsoptimierung von Flugzeugflügeln . . . . . . . . . . . . . . 143

M. Gröhlich, M. Böswald, Deutsches Zentrum für Luft- und Raumfahrt e.V.,

Institut für Aeroelastik, Göttingen;

R. Bauer, Universität Kassel

\section{> Schwingungsphänomene: Wälzlager in Antriebssystemen}

Simulation von Geräusch und Vibration in komplexen Systemen mit realen Wälzlagern. . . . . 157 H. Grillenberger, J. Fischer, Schaeffler Technologies AG \& Co KG, Herzogenaurach

Räumlich aufgelöste Wälzlagermodellierung für die elastische Mehrkörpersimulation . . . . . 169 S. Noack, M. Andersch, M. Beitelschmidt, Professur für Dynamik und Mechanismentechnik, Institut für Festkörpermechanik, Technische Universität Dresden

Detektion und Bewertung von Wälzlagerschäden mittels der Statistik höherer Ordnung . . . . 183 A. Trapp, J, Ochs, P. Wolfsteiner, Fakultät 03 für Maschinenbau, Fahrzeugtechnik, Flugzeugtechnik, Hochschule München 


\section{Condition Monitoring und KI-Methoden}

Extraktion und Selektion geeigneter Merkmale für die Restlebensdauerprognose von technischen Systemen trotz aleatorischen Unsicherheiten . . . . . . . . . . . . . . . . . . . . 197

0 . Kennedy Aimiyekagbon, A. Bender, W. Sextro, Universität Paderborn, Fakultät für Maschinenbau, Dynamik und Mechatronik, Paderborn

Mit Künstlicher Intelligenz Schwingungen / Akustik / NVH in Antriebssträngen vorhersagen und nachhaltig reduzieren - Praxisbeispiel aus der Industrie 4.0 . . . . . . . . . . . . . 211 F. Thurner, Contech Software \& Engineering GmbH, Fürstenfeldbruck

Mit Schwingungsanalyse Produktqualität und Produktionsprozesse in Echtzeit überwachen - Einsatz eines Softwaretools bei der Zahnradfertigung . . . . . . . . . . . . .223 5. Moschkau, Schaeffler Digital Solutions GmbH, Chemnitz

\section{$>$ Systemidentifikation}

Zustandsschätzung in dynamisch beanspruchten Strukturen am Beispiel von Windenergieanlagen . .

J. Luthe, J. Ehret, Y. Su, C. Woernle, Universität Rostock, Lehrstuhl für Technische Mechanik/

Dynamik, Rostock;

J. Zierath, W2E Wind to Energy GmbH, Rostock

Virtueller Sensor zur Überwachung von Schwingungen an spanenden Werkzeugmaschinen. .245 F. Dörrer, A. Otto, M. Kolouch, S. Ihlenfeldt, Fraunhofer-Institut für Werkzeugmaschinen und Umformtechnik IWU, Chemnitz

Anwendung einer Homotopie-Optimierungs-Methode zur Identifikation der Modellparameter eines Roboterprototyps . . . . . . . . . . . . . . . . . . . . . . . 259 Y. Luo, U. J. Römer, S. Riegraf, A. Fidlin, Institut für Technische Mechanik, Karlsruher Institut für Technologie, Karlsruhe;

M. Zirkel, L. Zentner, Fachgebiet Nachgiebige Systeme, Technische Universität Ilmenau, Ilmenau 


\section{Messmethoden und Identifikation}

Wasserfalldiagramme zur Interpretation von Rotorschwingungen - Theorie und Messung am Beispiel eines Lüfters . . . . . . . . . . . . . . . . . . . . . . . . 273 0. Zobel, M. Kreutz, T. Thümmel, Lehrstuhl für Angewandte Mechanik (Leitung: Prof. Rixen), Technische Universität München

Schwingungsverhalten von Zahnradgetrieben - Vibration behavior of gear transmissions . . . 285 A. Baumann, AVL Deutschland GmbH, Mainz-Kastel \& ZG TriboTech, Waiblingen

Bestimmung von dynamischen Eigenschaften bei Elastomerbauteilen mit Kompensation von Laufzeitunterschieden der Messsignale . . . . . . . . . . . . . . . . . . . . . . 303 T. Roth, T. Fürstner, M. Kröger, Institut für Maschinenelemente, Konstruktion und Fertigung, Technische Universität Bergakademie Freiberg;

B. Juretzki, IME Aachen GmbH Institut für Maschinenelemente und Maschinengestaltung

\section{$>$ Nichtlineare Systeme}

Identifikation nichtlinearer Strukturen - Vergleichbarkeit von Random- und Gleitsinus-Anregung . . . . . . . . . . . . . . . . . . . . . . . . . . . . . 317 M. Tang, M. Böswald, Y. Govers, Institut für Aeroelastik, Deutsches Zentrum für Luft- und Raumfahrt (DLR), Göttingen

Aktive trockene Reibungselemente in Tilgerkonfigurationen . . . . . . . . . . 333 J. A. Aramendiz, A. Fidlin, Karlsruher Institut für Technologie, Karlsruhe

\section{Plenarvortrag}

Über nichtglatte Dynamik . . . . . . . . . . . . . . . . . . . . . . . . . . . . . . 345

F. Pfeiffer, Angewandte Mechanik, Technische Universität, Garching 


\section{Ingenieure wollen immer alles ganz genau wissen. Wie wär's mit einem E-Paper- oder Zeitungs-Abo?}

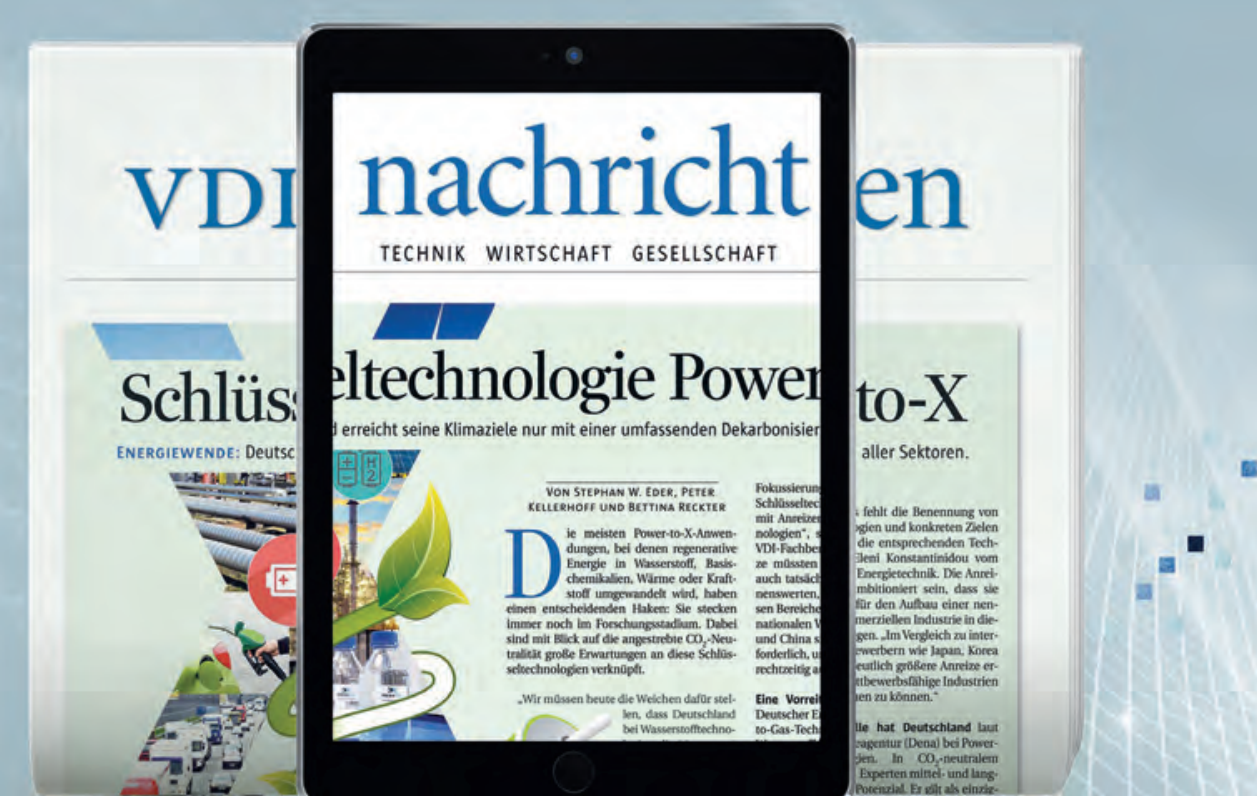

Mehr Meinung. Mehr Orientierung. Mehr Wissen.

Wesentliche Informationen zu neuen Technologien und Märkten. Das bietet VDI nachrichten, Deutschlands meinungsbildende Wochenzeitung zu Technik, Wirtschaft und Gesellschaft, den Ingenieuren. Sofort abonnieren und lesen.

Donnerstagabends als E-Paper oder freitags als Zeitung.

Jetzt abonnieren: Leser-Service VDI nachrichten, 65341 Eltville Telefon: +49 6123 9238-201, Telefax: +49 6123 9238-244, vdi-nachrichten@vuservice.de 


\section{Vorwort}

Sehr geehrte Damen und Herren,

moderne technische Produkte müssen hohe Anforderungen in Ihrem Festigkeits-, Schwingungs- und Geräuschverhalten erfüllen. Schwingungen und akustische Emissionen können dabei mitunter erwünscht sein - häufig sollen sie jedoch vermindert oder vermieden werden, um den Komfort, die Haltbarkeit oder die Betriebssicherheit zu steigern. Schwingungstechnik ist ein übergreifendes Thema, das in vielen verschiedenen Branchen präsent ist.

Lösungen zu Modellbildung und -validierung, Berechnung, Messung, Bewertung, Beeinflussung und Prognose können über die Grenzen von einzelnen Fachdisziplinen hinweg angewendet werden.

Die Behandlung der Phänomene sollte dabei bereits im Konstruktions- und Entwicklungsprozess beginnen. Dabei sind komplexe strukturdynamische und akustische Aufgaben zu lösen. Mit geeigneter Modellbildung und modernen Berechnungsverfahren können hierbei die Entstehung, die Ausbreitung und die Wirkung von Schwingungen und akustischen Auswirkungen vorhergesagt und bei Bedarf beeinflusst werden. Ist das technische System realisiert, kann das dynamische Verhalten experimentell erfasst werden. Des Weiteren können Maßnahmen zur strukturellen und akustischen Schwingungsbeeinflussung und -minderung angestoßen oder die Ergebnisse zur Überwachung sowie Identifikation des Zustands und der Prädiktion des Verhaltens einzelner Bauteile und/oder ganzer Systeme genutzt werden.

Auf allen genannten Gebieten findet sowohl in der Forschung als auch in der industriellen Praxis eine kontinuierliche Weiterentwicklung statt.

Die 3. VDI-Fachtagung Schwingungen bringt deshalb Experten aus Industrie und Forschung zusammen, um sich interdisziplinär über neue Verfahren und Erkenntnisse zu informieren und Erfahrungen auszutauschen.

Der vorliegende Tagungsband enthält alle Manuskripte der Referenten/-innen. Die Tagungsleiter und die Mitglieder des Programmausschusses danken allen, die beim Gelingen der Veranstaltung mitgewirkt haben. 
Wir freuen uns, Sie vom 16. bis 17. November 2021 auf der Festung Marienberg in Würzburg begrüßen zu dürfen und freuen uns auf interessante Vorträge und Gespräche mit allen Teilnehmern/-innen.

Im Namen des Programmausschusses

Ihre Tagungsleiter

Prof. Dr.-Ing. Michael Beitelschmidt, Professur Dynamik und Mechanismentechnik, Institut für Festkörpermechanik, Fakultät Maschinenwesen, Technische Universität Dresden

Dr.-Ing. Hans-Willi Keßler, Geschäftsführer, Schaeffler Monitoring Services GmbH, Herzogenrath

Dr.-Ing. Carsten Schedlinski, Geschäftsführer, ICS Engineering GmbH, Dreieich 